\title{
O impacto do isolamento social da COVID-19 na auto percepção da saúde geral e emocional de brasileiros
}

The impact of COVID-19 isolation on general and emotional health self-perception of Brazilians El impacto del aislamiento social de COVID-19 en la autopercepción de la salud general y emocional de los brasileños

Recebido: 16/12/2021 | Revisado: 22/12/2021 | Aceito: 28/12/2021 | Publicado: 06/01/2022

Priscila Feliciano de Oliveira

ORCID: https://orcid.org/0000-0002-6443-6167 Universidade Federal de Sergipe, Brasil

E-mail: priscila@academico.ufs.br

Amanda Souza de Jesus

ORCID: https://orcid.org/0000-0003-2243-8858 Universidade Federal de Sergipe, Brasil E-mail: amandasouzafga@gmail.com

Anderson Barbosa Sá Oliveira

ORCID: https://orcid.org/0000-0003-1526-2225 Universidade Federal de Sergipe, Brasil

E-mail: anderson_barbosa98@outlook.com

Swyanne Vitória Rodrigues dos Santos

ORCID: https://orcid.org/0000-0001-6500-8618 Universidade Federal de Sergipe, Brasil E-mail: swyanne_vitoria@hotmail.com

Aparecida Grasiele de Lima Silva

ORCID: https://orcid.org/0000-0002-0053-7567 Universidade Federal de Sergipe, Brasil E-mail: aparecidagrasielepf@gmail.com

Gabriele Sousa de Oliveira

ORCID: https://orcid.org/0000-0003-0908-5824 NASF da Prefeitura Municipal de Ribeirópolis, Brasil E-mail: gabriele.2012.1@icloud.com Oscar Felipe Falcão Raposo

ORCID: https://orcid.org/0000-0002-3110-7383 Universidade Federal de Sergipe, Brasil E-mail: oscar.raposo@academico.ufs.br

Raphaela Barroso Guedes-Granzoti

ORCID: https://orcid.org/0000-0002-9064-439X Universidade Federal de Sergipe, Brasil E-mail: raphaelabgg@gmail.com

\begin{abstract}
Resumo
A fim de proteger a população da COVID-19 e conter a disseminação do vírus foi proposto o isolamento social. Porém, o confinamento e as medidas restritivas de circulação geraram impactos negativos na população, no que diz respeito principalmente à saúde e ao aspecto emocional. Objetivo: Analisar o impacto do isolamento social da COVID-19 na autopercepção da saúde geral e emocional de brasileiros. Metodologia: Fizeram parte deste estudo 660 participantes, sendo a coleta realizada de agosto a setembro de 2020 durante a pandemia da Covid -19. Foi aplicado um questionário por meio eletrônico idealizado pelos pesquisadores com 26 perguntas fechadas de múltipla escolha (dados de identificação, saúde em geral e aspectos emocionais). Resultados: Média de idade de 36 anos, sendo $80 \%$ do gênero feminino, residentes de todos os estados brasileiros. 36,2\% trabalharam em home office e 17,4\% perderam o emprego durante a pandemia. $50,7 \%$ cumpriram o isolamento social à risca. Dentre os aspectos emocionais a ansiedade $(\mathrm{p}=0,083)$ e estresse $(\mathrm{p}=0,014)$ foram as mais destacadas, além de existir mudança significativa na alimentação $(\mathrm{p}=0,017)$ e presença de zumbido $(\mathrm{p}=0,070)$. Dos sujeitos positivos para COVID-19 $(\mathrm{n}=73)$ os dados foram significativos para estresse $(p=0,017)$, presença de zumbido $(p=0,038)$ e tontura $(p=0,000)$. Conclusão: Concluiu-se que o isolamento social foi um fator de risco para aumento do estresse, mudança de hábitos alimentares e aparecimento de zumbido, além de uma tendencia maior para ansiedade; e que a infecção por COVID-19 foi um fator de risco para o estresse e para o surgimento de tontura e zumbido entre os participantes.
\end{abstract}

Palavras-chave: COVID-19; Isolamento social; Saúde pública; Distanciamento físico. 


\begin{abstract}
In order to prevent COVID 19 spread it was proposed social isolation. However, strong social straining and social distance have raised negative impacts on the population, mainly about emotional and general health aspects. Objective: To analyze the impact of COVID-19 isolation on general and emotional health self-perception of Brazilians. Methodology: 660 participants took part of an online survey and the collection was carried out from August to September 2020 during the COVID-19 pandemic. The online survey was designed with 26 closed multiple-choice questions (identification data, general health and emotional aspects) by the researchers Results: The average age was 36 years, $80 \%$ were female and residents of all Brazilian states. $36.2 \%$ worked in a home office and $17.4 \%$ lost their jobs during the COVID-19 pandemic. Only 50.7\% carried out social isolation. Among the emotional aspects, anxiety $(\mathrm{p}=0.083)$ and stress $(\mathrm{p}=0.014)$ were the most highlighted, therefore there were a significant change in diet $(\mathrm{p}=0.017)$ and the presence of tinnitus $(p=0.070)$. The positive COVID-19 test $(n=73)$ were significant for stress $(p=0.017)$, presence of tinnitus $(\mathrm{p}=0.038)$ and dizziness $(\mathrm{p}=0.000)$. Conclusion: It was concluded that social isolation was a risk factor for increased stress, change in eating habits and the onset of tinnitus, in addition a greater tendency for anxiety; and COVID-19 infection was a risk factor for stress and for the onset of dizziness and tinnitus among the participants.

Keywords: COVID-19; Social isolation; Public health; Physical distancing.
\end{abstract}

\title{
Resumen
}

Con el fin de proteger a la población de COVID-19 y contener la propagación del virus, se propuso el aislamiento social. Sin embargo, el confinamiento y las medidas restrictivas de movimiento generaron impactos negativos en la población, principalmente en lo que respecta a la salud y el aspecto emocional. Objetivo: Analizar el impacto del aislamiento social de COVID-19 en la autopercepción de la salud general y emocional de los brasileños. Metodología: En este estudio participaron 660 participantes, y la recolección se realizó de agosto a septiembre de 2020 durante la pandemia COVID19. Se aplicó un cuestionario electrónico diseñado por los investigadores con 26 preguntas cerradas de opción múltiple (datos de identificación, salud general y aspectos emocionales). Resultados: Edad promedio de 36 años, $80 \%$ mujeres, residentes de todos los estados brasileños. El 36,2\% trabajaba en una oficina en casa y el 17,4\% perdió su empleo durante la pandemia. El 50,7\% cumplió al pie de la letra el aislamiento social. Entre los aspectos emocionales, la ansiedad $(\mathrm{p}=0,083)$ y el estrés $(\mathrm{p}=0,014)$ fueron los más destacados, además de un cambio significativo en la dieta ( $\mathrm{p}$ $=0,017)$ y la presencia de acúfenos $(\mathrm{p}=0,070)$. De los sujetos COVID-19 positivos $(\mathrm{n}=73)$ los datos fueron significativos para estrés $(p=0.017)$, presencia de tinnitus $(p=0.038)$ y mareos $(p=0.000)$. Conclusión: Se concluyó que el aislamiento social era un factor de riesgo para el aumento del estrés, el cambio de hábitos alimentarios y la aparición de acúfenos, además de una mayor tendencia a la ansiedad; y que la infección por COVID-19 era un factor de riesgo para el estrés y para la aparición de mareos y acúfenos entre los participantes.

Palabras clave: COVID-19; Aislamiento social; Salud pública; Distanciamiento físico.

\section{Introdução}

Em 11 de março de 2020, devido à rápida disseminação geográfica da COVID-19, doença causada pelo novo coronavírus (Sars-Cov-2) a Organização Mundial de Saúde (OMS) declarou estado de pandemia, sendo que atualmente o Brasil é o segundo país com maior número de óbitos causados pelo novo coronavírus, registrando mais de 600 mil mortes (World Health Organization, 2020a, 2020b).

Como medida de proteção e com o intuito de controlar a disseminação do vírus foi adotado em diversos países o isolamento social, e em alguns casos o "lockdown" como uma forma mais drástica de isolamento social proibindo inclusive a circulação de pessoas nas ruas. Diante dessa nova realidade observou-se uma relação positiva com a diminuição dos casos de indivíduos infectados em diversos países (Lau et al., 2020; Sjödin et al., 2020). Porém, o confinamento, as medidas restritivas de circulação em ambientes externos e o fechamento de todos os estabelecimentos não essenciais causaram uma ruptura das interações sociais, da economia e principalmente do aspecto emocional (Ammar, Chtourou, et al., 2020).

Diante da ruptura social, durante a pandemia da COVID-19 a população tentou manter estável a saúde mental, entretanto as medidas de enfrentamento para evitar a circulação do coronavírus afetou significativamente as relações familiares, sendo assim, as inovações tecnológicas foram as opções utilizadas pela população para aproximar os entes queridos (Ammar, Chtourou, et al., 2020). Acerca dessa nova era digital foi notável o avanço das plataformas virtuais e a telemedicina aos poucos foi sendo implantada e se tornou uma medida essencial para fazer o acompanhamento dos pacientes em estágio inicial da COVID-19 a fim de evitar o colapso na saúde (Wosik et al., 2020), bem como foi introduzida a telepsiquiatria para dar assistência aos sujeitos que necessitavam suporte relacionado a saúde mental (Di Carlo et al., 2021). Entretanto, mesmo com as mais diversas possibilidades 
vivenciadas pela nova era digital, esta não foi capaz de suprir a demanda da população acerca do convívio social, o qual gerou uma diminuição significativa no bem estar da população (Ammar, Chtourou, et al., 2020) que desencadeou sintomas como pânico, ansiedade, paranoia, comportamentos compulsivos, angústia e depressão (Dubey et al., 2020).

Outrossim, as pessoas infectadas não consideradas graves foram aconselhadas a fazer o tratamento em casa cumprindo a risca o distanciamento social, sendo que o recomendado era ficar totalmente isolado, sem contato com o mundo exterior. Tal ação impactou negativamente na saúde mental tanto do sujeito que testou positivo para COVID-19 quanto para os familiares, os quais conviviam com o medo de contrair a doença e de até mesmo perder o ente querido, aumentando assim o estresse e a ansiedade durante o período da quarentena (Khan et al., 2020). Observa-se ainda que existem sintomas considerados manifestações clínicas da COVID-19, como a tontura e zumbido (Mao et al., 2020; Saniasiaya \& Kulasegarah, 2021a; Viola et al., 2020), porém ainda não há relação destes com o isolamento social, sendo que são doenças raramente investigadas (Viola et al., 2021), e estudos acerca desta temática precisam ser desenvolvidos, uma vez que podem agravar o quadro emocional do seu portador (Pattyn et al., 2016) .

Ademais, a crise econômica foi outro fator que agravou os problemas emocionais, uma vez que houve a diminuição da produção de diversos setores como agricultura, turismo, petróleo, alimentício entre outros (Nicola et al., 2020); e a tão temida demissão se tornou realidade entre os familiares (Lesser \& Nienhuis, 2020; Matsungo \& Chopera, 2020). Diante das demandas sócio econômicas, as questões emocionais se tornaram evidentes durante a pandemia e estas manifestações psiquiátricas foram nomeadas "coronofobia", as quais podem perpetuar pós surto da COVID-19 (Dubey et al., 2020).

Sendo assim, é de extrema importância estudos voltados a saúde geral em época de isolamento social da COVID-19, uma vez que podem ser desencadeadas ou agravadas por problemas emocionais aliados as questões socioeconômicas, de interação social que podem culminar em depressão. Diante do exposto o objetivo desta pesquisa foi analisar o impacto do isolamento social da COVID-19 na autopercepção da saúde geral, emocional e laborativa de brasileiros.

\section{Metodologia}

Trata-se de um estudo observacional, transversal e analítico, aprovado pelo Comitê de Ética em Pesquisa (CAAE: 34932120.6.0000.5546). Todos os participantes assinaram de forma digital o Termo de Consentimento Livre e Esclarecido e receberam automaticamente uma via deste termo.

Fizeram parte desse estudo 660 participantes que foram recrutados por meio de divulgação da pesquisa via e-mail, WhatsApp e por redes sociais do Brasil. A participação ocorreu de forma remota, por meio da plataforma digital Google formulários ${ }^{\mathrm{R}}$. Foram incluídos indivíduos maiores de 18 anos, de qualquer gênero e residentes no Brasil. Foram excluídos os participantes que não preencheram todos os dados do questionário.

A coleta de dados foi realizada nos meses de agosto e setembro de 2020 e o instrumento utilizado foi um questionário de autopercepção idealizado pela pesquisadora responsável com 26 perguntas fechadas de múltipla escolha. Para elaboração das perguntas foi utilizado o modelo Total Design Method, o qual aperfeiçoa o número de respostas sem o preenchimento em tempo real com a presença do pesquisador e estipula regras para a elaboração do questionário (uso de perguntas fechadas; sem uso de questões vagas, muito precisas ou tendenciosas; sem muita exigência das perguntas para obtenção da resposta; agrupamento das questões quanto à similaridade de assuntos) (Dillman et al., 2009). Os aspectos abordados pelo instrumento foram: identificação (gênero, idade, estado de residência no Brasil), fatores emocionais atuais, saúde em geral (COVID-19, doenças da coluna cervical e metabólicas) e aspectos relacionado a tontura e zumbido (frequência e gatilho).

Foi realizada análise de forma descritiva e inferencial e utilizado o programa Statistical Package for Social Sciences versão 20 (SPSS, Chicago, USA) e a mesma. A descrição das variáveis qualitativas nominais foi realizada por meio de frequência 
relativa e absoluta. A descrição das variáveis quantitativas foi realizada por meio de medidas de variabilidade (desvio-padrão), tendência central (média e mediana) e posição (mínimo, máximo, primeiro quartil e terceiro quartil).

Para análise inferencial dos resultados primeiramente foi testada a normalidade da amostra por meio do teste de Smirnov Komorog. Na comparação entre dois grupos, sob influência de um único fator de variação foi realizado o teste Exato de Fischer. Consideramos estatisticamente significante se ( $\mathrm{p}$-valor $\leq 0,05$ ), e tendência de ser significante se o ( $\mathrm{p}$-valor $\leq 0,10$ ). A variável dependente foi o isolamento social e diagnóstico positivo para COVID-19. As independentes foram os aspectos emocionais, financeiro, nutricional, atividade física, tontura e zumbido.

\section{Resultados}

Grande parte dos participantes era do gênero feminino (80,3\%), com média de idade de 35,99 $(14,29)$ anos, e com representantes residentes em todos os estados brasileiros, sendo a maioria do estado de Sergipe (55\%), seguido por São Paulo $(17,3 \%)$ e Bahia (8,3\%). A tabela 1 apresenta o perfil dos participantes quanto à atividade laborativa que exerciam, se pertenciam ou não ao grupo de risco e se estavam realizando atividade física durante a pandemia da COVID-19.

Tabela 1. Distribuição dos participantes quanto à atividade laborativa, grupo de risco quanto às comorbidades e realização de atividade física durante a pandemia da COVID-19.

\begin{tabular}{|c|c|c|c|}
\hline Atividade laborativa & $\mathbf{N}$ & $(\%)$ & \\
\hline Conseguiu um trabalho/oportunidade durante a pandemia & 19 & $(2,9)$ & \\
\hline Continuou em casa, pois não trabalhava antes da pandemia & 178 & $(27,0)$ & \\
\hline Continuou trabalhando normalmente & 133 & $(20,2)$ & \\
\hline Ficou em casa sem trabalhar (autônomo/empresário) & 101 & $(15,3)$ & \\
\hline Ficou trabalhando em home office & 215 & $(32,6)$ & \\
\hline Foi demitido e fiquei em casa & 14 & $(2,1)$ & \\
\hline Comorbidades & $\mathbf{N}$ & $(\%)$ & P Valor \\
\hline Obesidade & 52 & $(7,87)$ & - \\
\hline Pressão arterial elevada & 86 & $(13,0)$ & - \\
\hline Colesterol elevado & 101 & $(15,30)$ & - \\
\hline Asma & 37 & $(5,60)$ & - \\
\hline Bronquite & 11 & $(1,60)$ & - \\
\hline Diabetes & 24 & $(3,63)$ & - \\
\hline Doenças cardíacas & 18 & $(2,72)$ & $0,002 *$ \\
\hline Rinite & 272 & $(41,2)$ & - \\
\hline Sinusite & 161 & $(24,4)$ & $0,097^{+}$ \\
\hline \multirow[t]{2}{*}{ Realização de atividade física na pandemia } & $\underset{\mathbf{n}(\%)}{\operatorname{Sim}}$ & $\begin{array}{l}\text { Não } \\
\text { n (\%) }\end{array}$ & P Valor \\
\hline & $208(53,5)$ & $181(46,5)$ & $0,000^{*}$ \\
\hline
\end{tabular}

Análise estatística: Teste exato de Fischer. ${ }^{*} \mathrm{p}<0,05{ }^{+} \mathrm{p}<0,10$. Fonte: Dados da pesquisa.

Em cumprimento ao decreto dos órgãos governamentais de distanciamento social observou-se que 50\% (330) dos participantes relataram realizá-lo à risca e permaneceram em isolamento social nas suas residências. A Tabela 2 demonstra que os participantes que cumpriram o isolamento social apresentaram respostas significativamente superior quanto a presença de estresse $(p=0,014)$ e a mudança de hábitos alimentares $(p=0,017)$ e uma tendência maior para ansiedade $(p=0,017)$ e presença de zumbido $(\mathrm{p}=0,070)$. Diante de sintomas emocionais verificou-se que $17 \%$ dos entrevistados relataram que durante a pandemia se consultaram com psiquiatra, sendo que 31,5\% não fizeram, mas gostariam de ter feito a consulta com especialista. Foi observado também uma mudança significativa dos hábitos alimentares entre as pessoas que cumpriram o isolamento social e aumento significativo de zumbido $(\mathrm{p}=0,007)$ nos participantes que relataram cumprir o isolamento social comparados aos que não realizaram o isolamento. 
Tabela 2: Análise dos aspectos emocionais, hábitos alimentares e presença de sintomas auditivos vestibulares nos participantes que cumpriram e não cumpriram o isolamento social.

\begin{tabular}{rcccc}
\hline & & Cumpriu o isolamento social & \\
\hline Aspectos emocionais & & $\begin{array}{c}\text { Sim } \\
\mathbf{n}(\boldsymbol{\%})\end{array}$ & $\begin{array}{l}\text { Não } \\
\mathbf{n}(\boldsymbol{\%})\end{array}$ & p Valor \\
\hline & Ansiedade & $245(74,2)$ & $228(69,1)$ & $0,083^{+}$ \\
\hline Tristeza & $159(48,2)$ & $153(46,4)$ & 0,348 \\
\hline Estresse & $216(65,5)$ & $243(73,6)$ & $0,014^{*}$ \\
\hline Insônia & $149(45,2)$ & $136(41,2)$ & 0,173 \\
\hline Angústia & $170(51,5)$ & $169(51,2)$ & 0,500 \\
\hline Medo & $143(43,3)$ & $155(40,9)$ & 0,291 \\
\hline Mudança dos hábitos alimentares & $195(59,1)$ & $167(50,6)$ & $0,017^{*}$ \\
\hline
\end{tabular}

Análise estatística: Teste exato de Fischer. ${ }^{*} \mathrm{p}<0,05^{+} \mathrm{p}<0,10$. Fonte: Dados da pesquisa.

Dentre os participantes, 73 relataram testagem positiva para COVID-19 e apresentaram um nível significativamente maior de estresse $(p=0,017)$ (Tabela 3$)$, assim como relataram o aparecimento de zumbido $(p=0,038)$ e tontura $(p=0,000)$ de forma significativa após a infecção por COVID-19.

Tabela 3. Análise dos aspectos emocionais, hábitos alimentares e presença de sintomas auditivos vestibulares nos participantes que testaram positivo ou não para COVID-19.

\begin{tabular}{|c|c|c|c|}
\hline \multirow[b]{2}{*}{ Aspectos emocionais } & \multicolumn{2}{|c|}{ Testou positivo para COVID-19 } & \multirow[b]{2}{*}{ p Valor } \\
\hline & $\begin{array}{c}\text { Sim } \\
\mathbf{n}(\%)\end{array}$ & $\begin{array}{c}\text { Não } \\
\text { n (\%) }\end{array}$ & \\
\hline Ansiedade & $53(72,6)$ & $74(66,7)$ & 0,246 \\
\hline Tristeza & $32(43,8)$ & $49(44,1)$ & 0,544 \\
\hline Estresse & $54(74,0)$ & $64(57,7)$ & $0,017 *$ \\
\hline Insônia & $31(42,5)$ & $45(40,5)$ & 0,457 \\
\hline Angústia & $43(58,9)$ & $53(47,7)$ & 0,091 \\
\hline Medo & $34(46,6)$ & $40(36,0)$ & 0,102 \\
\hline Mudança dos hábitos alimentares & $39(53,4)$ & $51(45,9)$ & 0,200 \\
\hline
\end{tabular}

Análise estatística: Teste exato de Fischer. ${ }^{*} \mathrm{p}<0,05^{+} \mathrm{p}<0,10$. Fonte: Dados da pesquisa.

\section{Discussão}

Os números de infectados pela COVID-19, no Brasil, sempre foi alarmante e do início da pandemia até a data da coleta dos dados, setembro de 2020 foi registrado um aumento de aproximadamente 800 mil novos casos, com uma média de mil mortes diárias, justificando a manutenção do isolamento do isolamento social (World Health Organization, 2020b). Portanto, durante o período de isolamento social o avanço tecnológico fez com que a população aderisse em massa as plataformas virtuais com o intuito de se aproximar dos entes queridos, vizinhos e amigos (Ammar, Chtourou, et al., 2020). Nesta vertente, com o intuito de abranger maior número de participantes na presente pesquisa, o estudo foi realizado por meio eletrônico (Ammar, Brach, et al., 2020; Ammar, et al., 2020; Beukes et al., 2020; Guimarães et al., 2021; Passos et al., 2020; Wang et al., 2020). A adesão na participação da pesquisa foi alta (660 pessoas), sendo que é possível observar respostas oriundas de todos os estados do território brasileiro, o que evidencia que o formato eletrônico foi capaz de potencializar a coleta de dados (Vieira et al., 2010). Com relação à faixa etária, observou-se predominância de respostas nas idades entre 20 e 29 anos, o que pode ser justificado pelos dados do Instituto Brasileiro de Geografia e Estatística (IBGE) o qual, reporta que esta faixa etária é considerada como maior usuária das redes sociais, sendo crescente o acesso à internet nestas idades em todos os estados brasileiros (Guimarães et al., 2021; IBGE, 2018b). 
No que diz respeito ao isolamento social verificou-se que apenas 50\% dos entrevistados seguiram o isolamento à risca. Apesar desta ser uma medida adotada por todos os países, a presente pesquisa reafirma que o Brasil apresentou baixa adesão nas medidas para enfrentamento da emergência de saúde pública do COVID-19 (Brasil, 2020). Estudos ainda reafirmam os dados da presente pesquisa, uma vez que no período da coleta de dados o IBGE relatou que 5,1 milhões de brasileiros interromperam as medidas de isolamento social mais rígida nas primeiras três semanas de setembro, perfazendo um total de $85,7 \%$ da população com adoção de medidas mais flexíveis (IBGE, 2018a). Acredita-se que as falhas relacionadas as medidas restritivas se devem a baixa adesão da população bem como a dificuldade da fiscalização dos órgãos governamentais (Khan et al., 2020).

Dentre os aspectos relacionados a saúde emocional, os entrevistados relataram angústia, tristeza, insônia, medo e presença de comportamento compulsivo. Observou-se ainda uma tendência significativa para a ansiedade e efeito positivo do isolamento relacionado ao estresse, dados semelhantes ao da literatura (Matsungo \& Chopera, 2020; Qiu et al., 2020; Wang et al., 2020). Constatou-se que esses aspectos surgiram após privação social imposta pela COVID-19, que mesmo com o avanço tecnológico das plataformas virtuais, estas não foram suficientes para diminuir os efeitos do distanciamento social e mesmo assim surgiu a ruptura do aspecto emocional (Ammar, Chtourou, et al., 2020).

É sabido que a presença de ansiedade e estresse podem levar ao seu portador depressão e podem vir acompanhadas da presença de pânico (Qiu et al., 2020; Wang et al., 2020), sendo assim é necessário o acompanhamento psicológico e/ou psiquiátrico, e até mesmo avaliação de uma equipe multidisciplinar (Ammar, et al., 2020; Smith \& Lim, 2020). Entretanto, observou-se que apenas 17\% dos entrevistados fizeram a busca efetiva de um profissional habilitado, número este reduzido. Talvez a baixa adesão se deve ao medo de contrair COVID-19 nos centros médicos de reabilitação. Em contrapartida para evitar o contágio nos ambientes hospitalares e centro médicos foi implementada medidas tecnológicas a favor da população, sendo elas: a telemedicina (Wosik et al., 2020) e telepsiquiatria (Di Carlo et al., 2021). Apesar da nova inovação tecnológica, esta não foi capaz de abranger grande parte da população e estudos reafirmam a necessidade da busca do atendimento, uma vez que os aspectos emocionais negativos desencadeados durante a pandemia devem ser tratados porque as sequelas podem se estender ao longo da vida, mesmo após término da quarentena (Pfefferbaum \& North, 2020) ..

Aliado aos fatores emocionais, a pandemia da COVID-19 gerou grande crise econômica mundial que levou a dificuldade financeira para a população como um todo. No período em que foi realizada a coleta de dados verificou-se que $15,3 \%$ dos participantes permaneceram em casa sem trabalho por serem autônomos/empresários e 2,1\% foram demitidos. A realidade brasileira com relação ao desemprego ou dificuldade em manter a família foi maior que nos estudos realizados no Zimbabwe (Matsungo \& Chopera, 2020), porém semelhante no Canada (Lesser \& Nienhuis, 2020). Vale ressaltar que a perda financeira também gera uma ruptura da vida, e pode ser um dos fatores que desencadeiam ou agravam os aspectos emocionais como raiva, medo e frustração (Brooks et al., 2020).

Com relação à alimentação observou-se uma alteração significativa dos hábitos alimentares entre os participantes que relataram cumprir o isolamento social. Acredita-se que tal fato tenha ocorrido em decorrência do fechamento dos estabelecimentos comerciais, sendo que os que continuaram abertos passaram a praticar aumento significativo do preço, diminuindo assim o poder de compra (Ammar, Chtourou, et al., 2020). Vale ressaltar ainda, que essa mudança de hábitos pode ter sido influenciada pelas dificuldades financeiras, uma vez que a família optou por mudar a alimentação para uma considerada menos saudável, a qual tinha preço mais atrativo do que os alimentos mais saudáveis (Matsungo \& Chopera, 2020). Ademias acredita-se que a alimentação com hábitos menos saudáveis, ou seja menor ingestão de vitaminas A,C,D,E e B, consideradas como importantes e atuantes no processo imunológico do ser humano (Sahin et al., 2020) podem ter tornado as pessoas mais suscetíveis ao contaminação da COVID-19, o que se torna um fator que pode explicar a alta disseminação da doença no Brasil.

Verificou-se o efeito do isolamento social na prática da atividade física, sendo que os participantes diminuíram a realização desta, dado comum em outros estudos (Ammar, et al., 2020; Ding et al., 2021; Lesser \& Nienhuis, 2020). Uma das 
explicações para este fator é o fechamento das áreas livres como parques, praças bem como das academias e também o aumento significativo do uso de dispositivos eletrônicos que distanciou a população da prática de atividade física (Ammar, et al., 2020). Estudos relatam a importância da prática efetiva de exercícios físicos a fim de beneficiar a saúde física, e a mental (Dwyer et al., 2020; Lesser \& Nienhuis, 2020), sendo assim acredita-se que os participantes da presente pesquisa poderiam ter diminuído os sintomas de ansiedade e estresse durante o período de isolamento social, se tivessem adotado os exercícios físicos como uma prática diária.

Sintomas auditivos vestibulares raramente são investigados (Viola et al., 2020), entretanto são queixas que podem surgir no período de isolamento social, bem como um sintoma da COVID-19. Desta maneira, o zumbido foi uma queixa auditiva significativa na presente pesquisa na população que realizou o isolamento social. Acredita-se que a presença de zumbido, de ordem psicossomática tenha ocorrido em decorrência da diminuição da interação social associada ao aumento do sentimento de solidão, as quais foram vivenciadas no período restritivo emergencial para o enfrentamento da COVID-19 (Beukes et al., 2020). Ademais a literatura reporta que a ansiedade, sintoma detectado na presente pesquisa, bem como a depressão são fatores que podem desencadear ou agravar o quadro de zumbido (Boecking et al., 2021; Pattyn et al., 2016).

Quanto aos participantes que testaram positivo para COVID-19, os dados foram significativos tanto para zumbido quanto para tontura, o que se justifica pelo fato destas alterações serem consideradas manifestações clínicas da COVID-19 (Li et al., 2020; Mao et al., 2020; Saniasiaya \& Kulasegarah, 2021b; Viola et al., 2020). No que diz respeito à tontura, 21,9\% dos entrevistados desencadearam a doença e 15,1\% agravaram. Achados na literatura relataram uma variabilidade de sujeitos com a presença deste sintoma que vai de 7 a 30\% (Baig et al., 2020; Q. Chen et al., 2020; T. Chen et al., 2020; Karadaş et al., 2020; Lo et al., 2020; Mi et al., 2020; Qiu et al., 2020; D. Wang et al., 2020; Z. Wang et al., 2020). A presença de tontura pode ser explicada em decorrência do processo inflamatório do sistema nervoso central (Karimi-Galougahi et al., 2020), uma vez que o Sars-Cov-2 entra no tecido neural pela circulação sanguínea, sendo que se liga aos receptores da enzima angiotensina 2 danificando a barreira hematoencefálica, desta maneira leva a um dano neural em decorrência do processo inflamatório (Baig et al., 2020; Wu et al., 2020). Com relação ao zumbido, 16,4\% desencadearam o sintoma auditivo pós COVID-19 e 13,7\% agravaram. A literatura reporta uma porcentagem mais alta que a da presente pesquisa de 20,0 a 23,2 de presença de zumbido após infecção pelo coronavírus (Freni et al., 2020; Viola et al., 2020). É sabido que as infecções virais podem causar prejuízos nas estruturas e no funcionamento do sistema nervoso central (Wu et al., 2020), e a presença do zumbido pode ser decorrente do neurotropismo e do poder neuroinvasivo da COVID-19 na região de cabeça e pescoço (Freni et al., 2020).

Em suma, o aparecimento do zumbido está associado não somente isolamento social, mas também à infecção pelo COVID-19, enquanto a tontura, a princípio está associada apenas à infecção por COVID-19. Tais achados corroboram as informações da literatura de que a pandemia tem implicações alarmantes para a saúde individual e coletiva e para o funcionamento social, emocional e auditivo vestibular da população em geral (Baig et al., 2020; Beukes et al., 2020; Pfefferbaum \& North, 2020).

Ademais, os dados apresentados mostram que a Pandemia COVID-19 gerou uma ocorrência elevada de estresse nos participantes que realizaram o isolamento social e nos que se infectaram com o novo coronavírus. Acredita-se que a mídia tenha sido um dos fatores geradores de estresse na população, uma vez que as constantes informações e "desinformações" da COVID19, apesar de manter a sociedade atualizada também gerava medo e estresse em relação ao contágio da doença (Gottlieb \& Dyer, 2020). Outrossim, o estresse em decorrência da testagem positiva da COVID-19 pode estar aliada ao medo de morrer, bem como ao neurotropismo da doença que gera um processo inflamatório do sistema nervoso central, o qual pode exacerbar as memórias traumáticas vivenciadas pelo contágio da doença, dificultando assim uma recuperação estável (Steardo et al., 2020).

Estudos avançados acerca das desordens auditivas vestibulares durante o período de isolamento social devem ser realizadas, sendo importante o uso das avaliações auditivas objetivas e não somente a aplicação de questionários. Além disso, 
outras limitações do presente estudo incluem a falta de informações sobre sintomas da saúde em geral e emocional dos participantes anteriores à pandemia e a utilização do meio digital para a coleta de dados que acaba por excluir os sujeitos que não possuem familiaridade/falta de habilidade com a tecnologia digital e impede que o participante tenha auxílio quando não compreende alguma pergunta, podendo fazer em algumas situações que ignore o preenchimento do questionário.

\section{Conclusão}

Apenas metade dos participantes realizaram o isolamento social durante a pandemia de COVID-19 e que este isolamento foi um fator de risco para aumento do estresse, mudança de hábitos alimentares e aparecimento de zumbido, além de uma tendência maior para ansiedade. E que a infecção por COVID-19 foi um fator de risco para o estresse e para o surgimento de tontura e zumbido entre os participantes.

Assim, torna-se urgente a implementação de estratégias e políticas públicas pós pandemia que possam orientar e tratar tanto os sintomas emocionais como da saúde em geral da população, entendendo estes sintomas como sequelas não só da infecção pelo vírus, mas como decorrentes das necessidades de mudanças e adaptações nas relações sociais.

\section{Referências}

Ammar, A., Brach, M., Trabelsi, K., Chtourou, H., Boukhris, O., Masmoudi, L., Bouaziz, B., Bentlage, E., How, D., Ahmed, M., Müller, P., Müller, N., Aloui, A., Hammouda, O., Paineiras-Domingos, L. L., Braakman-Jansen, A., Wrede, C., Bastoni, S., Pernambuco, C. S., \& Hoekelmann, A. (2020). Effects of COVID19 home confinement on eating behaviour and physical activity: Results of the ECLB-COVID19 international online survey. Nutrients, 12 (6), 1-13.

Ammar, A., Chtourou, H., Boukhris, O., Trabelsi, K., Masmoudi, L., Brach, M., Bouaziz, B., Bentlage, E., How, D., Ahmed, M., Mueller, P., Mueller, N., Hsouna, H., Aloui, A., Hammouda, O., Paineiras-Domingos, L. L., Braakman-Jansen, A., Wrede, C., Bastoni, S., \& Hoekelmann, A. (2020). Covid-19 home confinement negatively impacts social participation and life satisfaction: A worldwide multicenter study. Int J Environ Res Public Health, 17(17), 1-17.

Ammar, A., Mueller, P., Trabelsi, K., Chtourou, H., Boukhris, O., Masmoudi, L., Bouaziz, B., Brach, M., Schmicker, M., Bentlage, E., How, D., Ahmed, M., Aloui, A., Hammouda, O., Paineiras-Domingos, L. L., Braakman-Jansen, A., Wrede, C., Bastoni, S., Pernambuco, C. S., \& Hoekelmann, A. (2020). Psychological consequences of COVID-19 home confinement: The ECLB-COVID19 multicenter study. PloS One, 15(11), 1-12.

Baig, A. M., Khaleeq, A., Ali, U., \& Syeda, H. (2020, April 1). Evidence of the COVID-19 Virus Targeting the CNS: Tissue Distribution, Host-Virus Interaction, and Proposed Neurotropic Mechanisms. ACS Chem Neurosci, 11(7), 8.

Beukes, E., Baguley, D., Jacquemin, L., Lourenco, M., Allen, P., Onozuka, J., Stockdale, D., Kaldo, V., Andersson, G., \& Manchaiah, V. (2020). Changes in Tinnitus Experiences During the COVID-19 Pandemic. Front Public Health, 8(5), 1-13.

Boecking, B., Biehl, R., Brueggemann, P., \& Mazurek, B. (2021). Health-Related Quality of Life, Depressive Symptoms, Anxiety, and Somatization Symptoms in Male and Female Patients with Chronic Tinnitus. J Clin Med ., 10(13), 2798.

Brasil. (2020). No Lei No 13.979, de 6 de fevereiro de 2020. Dispõe sobre as medidas para enfrentamento da emergência de saúde pública de importância internacional decorrente do coronavírus responsável pelo surto de 2019. http://www.planalto.gov.br/ccivil_03/_ato2019-2022/2020/lei/L13979.htm

Brooks, S. K., Webster, R. K., Smith, L. E., Woodland, L., Wessely, S., Greenberg, N., \& Rubin, G. J. (2020). The psychological impact of quarantine and how to reduce it: rapid review of the evidence. Lancet, 395(10227), 912-920.

Chen, Q., Zheng, Z., Zhang, C., Zhang, X., Wu, H., Wang, J., Wang, S., \& Zheng, C. (2020). Clinical characteristics of 145 patients with corona virus disease 2019 (COVID-19) in Taizhou, Zhejiang, China. Infection, 48(4), 51.

Chen, T., Wu, D., Chen, H., Yan, W., Yang, D., Chen, G., Ma, K., Xu, D., Yu, H., Wang, H., Wang, T., Guo, W., Chen, J., Ding, C., Zhang, X., Huang, J., Han, M., Li, S., Luo, X., ... Ning, Q. (2020). Clinical characteristics of 113 deceased patients with coronavirus disease 2019: BMJ, 368, 1-28

Di Carlo, F., Sociali, A., Picutti, E., Pettorruso, M., Vellante, F., Verrastro, V., Martinotti, G., \& di Giannantonio, M. (2021). Telepsychiatry and other cuttingedge technologies in COVID-19 pandemic: Bridging the distance in mental health assistance. Int J Clin Pract ., 75(1),e13716

Dillman, D. A., Smyth, J. D., \& Christian, L. M. (2009). Internet, Mail, and Mixed-Mode Surveys: The Tailored Design Method. John Wiley and Sons.

Ding, K., Yang, J., Chin, M., Sullivan, L., Durstine, J., Violant-Holz, V., Demirhan, G., Oliveira, N., Popeska, B., Kuan, G., Khan, W., Dai, J., Xu, X., Mladenova, Z., Balasekaran, G., \& Smith, G. (2021). Physical Activity among Adults Residing in 11 Countries during the COVID-19 Pandemic Lockdown. International Journal of Environmental Research and Public Health, 18(13), 7056.

Dubey, S., Biswas, P., Ghosh, R., Chatterjee, S., Dubey, M. J., Chatterjee, S., Lahiri, D., \& Lavie, C. J. (2020). Psychosocial impact of COVID-19. Diabetes Metab Syndr, 14(5), 779-788.

Dwyer, M., Pasini, M., De Dominicis, S., \& Righi, E. (2020). Physical activity: Benefits and challenges during the COVID-19 pandemic. Sccand J Med Sci Sports ., 30(7), 1291-1294 
Freni, F., Meduri, A., Gazia, F., Nicastro, V., Galletti, C., Aragona, P., Galletti, C., Galletti, B., \& Galletti, F. (2020). Symptomatology in head and neck district in coronavirus disease (COVID-19): A possible neuroinvasive action of SARS-CoV-2. Am J Otolaryngol ., 41(5), 1-8.

Gottlieb, M., \& Dyer, S. (2020). Information and Disinformation: Social Media in the COVID-19 Crisis. Acad Emerg Med ., 27(7), 640-641.

Guimarães, V. H. A., de Oliveira-Leandro, M., Cassiano, C., Marques, A. L. P., Motta, C., Freitas-Silva, A. L., de Sousa, M. A. D., Silveira, L. A. M., Pardi, T. C., Gazotto, F. C., Silva, M. V., Rodrigues, V., Rodrigues, W. F., \& Oliveira, C. J. F. (2021). Knowledge about COVID-19 in Brazil: Cross-sectional web-based study. JMIR Public Health Surveill. , 7(1), e24756.

IBGE. (2018a). Cai para 33,8 milhões número de pessoas rigorosamente isoladas na pandemia. Censo Agro 2017. https://agenciadenoticias.ibge.gov.br/agencianoticias/2012-agencia-de-noticias/noticias/29126-cai-para-33-8-milhoes-numero-de-pessoas-rigorosamente-isoladas-napandemia?utm_source=covid19\&utm_medium=hotsite\&utm_campaign=covid_19

IBGE. (2018b). PNAD Contínua TIC 2017: Internet chega a três em cada quatro domicílios do país. Agência IBGE. https://agenciadenoticias.ibge.gov.br/agencia-sala-de-imprensa/2013-agencia-de-noticias/releases/23445-pnad-continua-tic-2017-internet-chega-a-tres-emcada-quatro-domicilios-do-pais

Karadaş, Ö., Öztürk, B., \& Sonkaya, A. R. (2020). A prospective clinical study of detailed neurological manifestations in patients with COVID-19. Neurol Sci , 41(8), 1991-1995.

Karimi-Galougahi, M., Naeini, A. S., Raad, N., Mikaniki, N., \& Ghorbani, J. (2020). Vertigo and hearing loss during the COVID-19 pandemic - is there an association?La vertigine ed l'ipoacusia durante la pandemia COVID-19 - esiste un nesso? Acta Otorhinolaryngol Ital, 1-3.

Khan, M., Adil, S. F., Alkhathlan, H. Z., Tahir, M. N., Saif, S., Khan, M., \& Khan, S. T. (2020). COVID-19: A Global Challenge with Old History, Epidemiology and Progress So Far. Molecules , 26(1), 39.

Khan, S., Siddique, R., Li, H., Ali, A., Shereen, M. A., Bashir, N., \& Xue, M. (2020). Impact of coronavirus outbreak on psychological health. J Glob Health, 10(1), 010331.

Lau, H., Khosrawipour, V., Kocbach, P., Mikolajczyk, A., Schubert, J., Bania, J., \& Khosrawipour, T. (2020). The positive impact of lockdown in Wuhan on containing the COVID-19 outbreak in China. J TravelMed, 27(3), taaa037.

Lesser, I., \& Nienhuis, C. (2020). The Impact of COVID-19 on Physical Activity Behavior and Well-Being of Canadians. Int J Environ Res Public Health, $17(11), 1-22$.

Li, L., Huang, T., Wang, Y., Wang, Z., Liang, Y., Huang, T., Zhang, H., Sun, W., \& Y, W. (2020). COVID-19 patients' clinical characteristics, discharge rate, and fatality rate of meta-analysis. J Med Virol ., 92(6), 83.

Lo, I. L., Lio, C. F., Cheong, H. H., Lei, C. I., Cheong, T. H., Zhong, X., Tian, Y., \& Sin, N. N. (2020). Evaluation of sars-cov-2 rna shedding in clinical specimens and clinical characteristics of 10 patients with COVID-19 in Macau. Int J Biol Sci ., 16(10), 707.

Mao, L., Jin, H., Wang, M., Hu, Y., Chen, S., He, Q., Chang, J., Hong, C., Zhou, Y., Wang, D., Miao, X., Li, Y., \& Hu, B. (2020). Neurologic Manifestations of Hospitalized Patients with Coronavirus Disease 2019 in Wuhan, China. JAMA Neurol , 77(6), 90.

Matsungo, T. M., \& Chopera, P. (2020). Effect of the COVID-19-induced lockdown on nutrition, health and lifestyle patterns among adults in Zimbabwe. BMJ Nutr Prev Health, 3(2), 12.

Mi, B., Chen, L., Xiong, Y., Xue, H., Zhou, W., \& Liu, G. (2020). Characteristics and early prognosis of covid-19 infection in fracture patients. J Bone Joint Surg Am ., 102(9), 8.

Nicola, M., Alsafi, Z., Sohrabi, C., Kerwan, A., Al-Jabir, A., Iosifidis, C., Agha, M., \& Agha, R. (2020). The socio-economic implications of the coronavirus pandemic (COVID-19): A review. Int J Surg . , 78, 93

Passos, L., Prazeres, F., Teixeira, A., \& Martins, C. (2020). Impact on mental health due to covid-19 pandemic: Cross-sectional study in portugal and brazil. Int J Environ Res Public Health, 17(18), 1-13.

Pattyn, T., Van Den Eede, F., Vanneste, S., Cassiers, L., Veltman, D. J., Van De Heyning, P., \& Sabbe, B. C. G. (2016). Tinnitus and anxiety disorders: A review. Hear Res, 333, 255-265.

Pfefferbaum, B., \& North, C. S. (2020). Mental Health and the Covid-19 Pandemic. N Engl J Med ., 383(6), 2.

Qiu, J., Shen, B., Zhao, M., Wang, Z., Xie, B., \& Xu, Y. (2020). A nationwide survey of psychological distress among Chinese people in the COVID-19 epidemic: Implications and policy recommendations. Gen Psychiatr, 33(2),e100213

Sahin, E., Orhan, C., Uckun, F. M., \& Sahin, K. (2020). Clinical Impact Potential of Supplemental Nutrients as Adjuncts of Therapy in High-Risk COVID-19 for Obese Patients. Front Nutr. , 7, 580504.

Saniasiaya, J., \& Kulasegarah, J. (2021a). Dizziness and COVID-19. Ear Nose Throat J ., 100(1), 29-30.

Sjödin, H., Wilder-Smith, A., Osman, S., Farooq, Z., \& Rocklöv, J. (2020). Only strict quarantine measures can curb the coronavirus disease (COVID-19) outbreak in Italy, 2020. Euro Surveill, 25(13), 2000280.

Smith, B., \& Lim, M. (2020). How the COVID-19 pandemic is focusing attention on loneliness and social isolation. Public Health Res Pract ., 30 (2), 3022008.

Steardo, L., Steardo, L., \& Verkhratsky, A. (2020). Psychiatric face of COVID-19. Transl Psychiatry ., 10(1), 1-12.

Vieira, H. C., Castro, A. E., \& Schuch Júnior, V. F. (2010). O uso de questionários via e-mail em pesquisas acadêmicas sob a ótica dos respondentes. XIII 
Research, Society and Development, v. 11, n. 1, e26711124818, 2022

(CC BY 4.0) | ISSN 2525-3409 | DOI: http://dx.doi.org/10.33448/rsd-v11i1.24818

SEMEAD: Seminários Em Administração, 1-13.

Viola, P., Ralli, M., Pisani, D., Malanga, D., Sculco, D., Messina, L., Laria, C., Aragona, T., Leopardi, G., Ursini, F., Scarpa, A., Topazio, D., Cama, A., Vespertini, V., Quintieri, F., Cosco, L., Cunsolo, E. M., \& Chiarella, G. (2020). Tinnitus and equilibrium disorders in COVID-19 patients: preliminary results. Eur Arch Otorhinolaryngol . 278(10), 1-6.

Wang, C., Pan, R., Wan, X., Tan, Y., Xu, L., Ho, C. S., \& Ho, R. C. (2020). Immediate psychological responses and associated factors during the initial stage of the 2019 coronavirus disease (COVID-19) epidemic among the general population in China. Int J Environ Res Public Health, 17(5), 1-25.

Wang, D., Hu, B., Hu, C., Zhu, F., Liu, X., Zhang, J., Wang, B., Xiang, H., Cheng, Z., Xiong, Y., Zhao, Y., Li, Y., Wang, X., \& Peng, Z. (2020). Clinical Characteristics of 138 Hospitalized Patients with 2019 Novel Coronavirus-Infected Pneumonia in Wuhan, China. JAMA, 323(11), 9.

Wang, Z., Yang, B., Li, Q., Wen, L., \& Zhang, R. (2020). Clinical features of 69 cases with coronavirus disease 2019 in Wuhan, China. Clin Infect Dis, 71(15), 77.

World Health Organization. (2020a). Novel Coronavirus (2019-nCoV) Situation Report - 10 (30 january 2020). https://www.who.int/docs/defaultsource/coronaviruse/situation-reports/20200130-sitrep-10-ncov.pdf?sfvrsn=d0b2e480_2

World Health Organization. (2020b). Weekly epidemiological update - 28 September 2020. https://www.who.int/publications/m/item/weekly-epidemiologicalupdate---28-september-2020

Wosik, J., Fudim, M., Cameron, B., Gellad, Z. F., Cho, A., Phinney, D., Curtis, S., Roman, M., Poon, E. G., Ferranti, J., Katz, J. N., \& Tcheng, J. (2020). Telehealth transformation: COVID-19 and the rise of virtual care. J Am Med Inform Assoc ., 27(6), 62.

Wu, Y., Xu, X., Chen, Z., Duan, J., Hashimoto, K., Yang, L., Liu, C., \& Yang, C. (2020, July 1). Nervous system involvement after infection with COVID-19 and other coronaviruses. Brain Behav Immun , 87, 18-22. 